\title{
DIVISION ALGEBRA COPRODUCTS OF INDEX $n$
}

\author{
MICHEL VAN DEN BERGH AND AIDAN SCHOFIELD
}

\begin{abstract}
Given a family of separable finite dimensional extensions $\left\{L_{i}\right\}$ of a field $k$, we construct a division algebra $n^{2}$ over its center which is freely generated over $k$ by the fields $\left\{L_{i}\right\}$.
\end{abstract}

\section{INTRODUCTION}

We fix a ground field $k$. This paper arose from the wish to understand what restrictions, if any, there are on the collections of algebraic elements in a division algebra of index $n$ over a field extension $K$ of $k$. The example of Cohn's work on skew field products suggested the approach of studying the ring coproduct of a family of field extensions $\left\{L_{i}: i \in I\right\}$ of $k$ modulo the identities of $n$ by $n$ matrices. This study is carried out in $\S 2$ subject to the results that are proved in $\S \S 3$ and 4 . We show that there is a division algebra that deserves the name of a division algebra coproduct of index $n$, and we describe its algebraic elements.

In order to prove that a prime polynomial identity algebra is a domain, it is usually necessary to find a large number of homomorphisms from the given algebra to division algebras of the correct index. In our case, the problem is to find a division algebra $D$ of index $n$ of center $K \supset k$ such that $L_{i} \otimes_{k} K$ embeds in $D$ as a $K$-algebra. We solve this problem in $\S 3$. Let $D$ be a central division algebra over $k$ of index and order $n$. Let $L$ be a separable extension of $k$ of dimension dividing $n$. We construct a field $K \supset k$ such that $L \otimes_{k} K$ embeds in the division algebra $D \otimes_{k} K$. The construction is geometric and the algebraic varieties we need are closely related to Brauer-Severi varieties.

One consequence of the above stated results is that there exist division algebras $D$ and $E$ of index $n$ over some field $K$ such that subfields of $D$ and $E$ are the same, but the subgroup of the Brauer group generated by the classes of $D$ and $E$ is isomorphic to $(\mathbf{Z} / n \mathbf{Z})^{2}$. The referee pointed out to us that $\mathbf{B}$. Fein has constructed such examples where $K$ is a number field [4].

The other problem left in $\S 2$ to be dealt with in $\S 4$ is the question of the polynomial identity degree of the ring coproduct of the fields $L_{i}$ amalgamating $k$ modulo the identities of $n$ by $n$ matrices. Is it really $n$ ? If there are only two fields $L_{1}$ and $L_{2}$ both of which are quadratic, the answer is no, because

Received by the editors October 5, 1989 and, in revised form, January 22, 1990.

1980 Mathematics Subject Classification (1985 Revision). Primary 14M15, 16A38, 16A39.

The first author supported by an NFWO-grant. 
the ring coproduct itself is known to have polynomial identity degree 2 [2]. However, in all other cases, we show that the answer is yes.

This is a consequence of the following result. We prove that if $L_{1}$ and $L_{2}$ are simple separable subalgebras of the central simple $k$-algebra $S$ of dimension $n^{2}$ over $k$, and, if either $L_{1}$ or $L_{2}$ has dimension larger than 2 over $k$, then there exists a unit $u \in S$ such that $L_{1}$ and $L_{2}^{u}$ generate $S$ as an algebra.

\section{NOTATION AND TERMINOLOGY}

We work over the ground field $k$. A field extension $K \supset k$ is said to be regular if $K \otimes_{k} \overline{\bar{k}}$ is a field where $\overline{\bar{k}}$ is the algebraic closure of $k$, alternatively, $K$ is the function field of an absolutely irreducible variety.

If $V$ is a vector space, $[V: k]$ is its dimension. If $D$ is a central division algebra over $k,[D: k]=n^{2}$, we say that $n$ is the index of $D$. The order of $D$ is the order of the class of $D,[D]$, in the Brauer group of $k$. We say that the index of a central simple algebra $S$ is the index of the division algebra similar to $S$; if $[S: k]=n^{2}$, we say that $S$ has P.I. degree $n$. Here P.I. stands for polynomial identity. If $R$ is a prime P.I. algebra, its P.I. degree is equal to that of its simple ring of fractions.

If $R$ is an algebra over $k, \operatorname{spec} R$ is the collection of prime ideals of $R$ equipped with the Zariski topology. If $R$ is prime and has P.I. degree $n$, the set of prime ideals $p$ such that $R / p$ has P.I. degree $n$ is open in the Zariski topology; it is denoted by $\operatorname{spec}_{n} R$. $R$ is said to be a Jacobson ring if any prime ideal is an intersection of maximal ideals. Any finitely generated P.I. algebra is Jacobson [9].

Given a collection of $k$-algebras $\left\{L_{i}: i \in I\right\}, *_{k} L_{i}$ is the ring coproduct of the algebras $L_{i}$ amalgamating $k$; it represents the functor $\prod_{i \in I} \operatorname{Hom}\left(L_{i},-\right)$ in the category of $k$-algebras.

If $R$ is an algebra, $(R)_{n}$ denotes $R / P_{n}$ where $P_{n}$ is the ideal generated by the evaluations of all polynomial identities of $n \times n$-matrices.

If $S$ is a central simple algebra, and $E \subset S$ is a subalgebra, $C_{S}(E)$ is its centralizer. $S^{*}$ is the group of units of $S$.

\section{THE DIVISION ALGEBRA COPRODUCT OF INDEX $n$}

The problem we shall be considering in this section is the following, given separable field extensions $\left\{L_{i}: i=1, \ldots, n\right\}$ of finite dimension dividing $n$ over the common subfield $k$, is there a "generic" division algebra of index $n$ over some field extension $K \supset k$ such that $D$ contains $L_{i}$ for $i=1$ to $m$.

A general discussion of this problem will clarify the notion of generic required.

Our first step in the construction of the generic division algebra is the ring $C=\left(*_{k} L_{i}\right)_{n}$; here $*_{k} L_{i}$ is the ring coproduct of the fields $L_{i}$, amalgamating the subfield $k$, modulo the identities of $n \times n$-matrices. In the case where there are just two fields $L_{1}$ and $L_{2} \supset k$ both of which are 2-dimensional, Bergman has the following result [2].

Theorem 2.1. $L_{1} *_{k} L_{2}$ is a principal domain of dimension 4 over its center which is isomorphic to a polynomial ring in one variable over $k$.

This has the consequence that two quadratic field extensions can only generate a division algebra of index 2 , and the division algebra of fractions of $L_{1} *_{k} L_{2}$ is 
a suitable candidate for a division algebra coproduct of index 2 of $L_{1}$ and $L_{2}$ over $k$. This is misleading in two respects. We shall see that if there are more than two fields, or if one of the dimensions of the extensions is larger than two, then they may generate division algebras of arbitrarily large index, and also we shall see that $\left(*_{k} L_{i}\right)_{n}$ is usually not prime. However, the first difference is useful for us, and the second arises because we have not been sufficiently specific.

Consider $C=\left(*_{k} L_{i}\right)_{n}$; we do not know whether the nil-radical of $C$ is 0 . However, it is not a worrying problem since we are interested in spec $C$, and, in particular, $\operatorname{spec}_{n} C$. One knows that $\operatorname{spec}_{n} C$ is an open subset of $\operatorname{spec} C$, and the results of $\S 4$ show that it is nonempty except in the case of two quadratic extensions. We shall assume from now on that we have excluded this case, since it has been completely dealt with by Theorem 2.1 . Our first aim is to find the minimal primes of $C$ that lie in $\operatorname{spec}_{n}(C)$. It is helpful to examine the prime ideals of $C \otimes_{k} \bar{k}$ where $\bar{k}$ is the separable closure of $k$, so we begin by describing the connection between $\operatorname{spec} C$ and $\operatorname{spec}\left(C \otimes_{k} \bar{k}\right)$.

Lemma 2.2. Let $\left\{A_{i}: i \in I\right\}$ be a family of $k$-algebras, and let $L \supset k$ be an extension of fields. Then $\left(*_{k} A_{i}\right)_{n} \otimes_{k} L \simeq\left(*_{L} A_{i} \otimes_{k} L\right)_{n}$.

Proof. They represent the same functor in the category of $L$-algebras satisfying the identities of $n$ by $n$ matrices.

Let $G$ be the Galois group of $\bar{k}$ over $k$. The relationship between $\operatorname{spec} C$ and $\operatorname{spec} \bar{C}$ is given by the following lemma.

Lemma 2.3. Let $A$ be an arbitrary k-algebra. Then $\operatorname{spec} A=\operatorname{spec}\left(A \otimes_{k} \bar{k}\right) / G$, that is, the points of $\operatorname{spec} A$ correspond to orbits of $G$ on $\operatorname{spec}\left(A \otimes_{k} \bar{k}\right)$. Further, $\operatorname{spec}_{n} A=\operatorname{spec}_{n}\left(A \otimes_{k} \bar{k}\right) / G$.

Proof. This is well known for finite Galois extensions, and the general case follows by a standard direct limit argument.

Now we face the problem of describing $\operatorname{spec}_{n}\left(C \otimes_{k} \bar{k}\right)$; we call $\bar{C}=C \otimes_{k} \bar{k}$, $\bar{L}_{i}=L_{i} \otimes_{k} \bar{k}$. Since $\bar{C}$ is a finitely generated P.I. algebra, it is a Jacobson ring [9], that is, all prime ideals are intersections of maximal ideals, which all have finite codimension in $\bar{C}$. So, we look at $\Omega_{n}(\bar{C})$, the collection of maximal ideals of P.I. degree $n$. These are represented by surjective $\bar{k}$-maps $\phi: \bar{C} \rightarrow M_{n}(\bar{k})$, and two maps represent the same point if and only if they are conjugate in $M_{n}(\bar{k})$. A map $\phi: \bar{C} \rightarrow M_{n}(\bar{k})$ is uniquely represented by a collection of maps $\phi_{i}: \bar{L}_{i} \rightarrow M_{n}(\bar{k})$. Since $\bar{k}$ is separably closed and each $L_{i} \supset k$ is separable, $\bar{L}_{i} \simeq \bar{k} e_{i 1} \times \cdots \times \bar{k} e_{i m_{i}}$ where $\left\{e_{i j}: i=1, \ldots, m\right.$, $\left.j=1, \ldots, m_{i}\right\}$ are idempotents. The maps $\phi_{i}: \bar{L}_{i} \rightarrow M_{n}(\bar{k})$ make $V=\bar{k}^{n}$ into an $\bar{L}_{i}$-module so we may associate to each $\phi: \bar{C} \rightarrow M_{n}(\bar{k})$, a collection of numbers $\left\{h_{i j}\right\}$ such that $\left[V\left(\phi\left(e_{i j}\right)\right): k\right]=h_{i j}$. These numbers satisfy the conditions $\sum_{j} h_{i j}=n$.

We consider $\bar{C}$ as a sheaf of rings over $\operatorname{spec}(Z(\bar{C}))$ where $Z(\bar{C})$ is the center of $\bar{C}$. The image of $\operatorname{spec}_{n} \bar{C}$ is an open subset $U$ of $\operatorname{spec} Z(\bar{C})$ and the restriction of $\bar{C}$ to $U$ is a sheaf of Azumaya algebras of index $n$. We restrict our attention to this sheaf of Azumaya algebras.

Theorem 2.4. Let $H=\left\{h_{i j}: i=1, \ldots, m, j=1, \ldots, m_{i}\right\}$ be a collection of numbers such that $\sum_{j} h_{i j}=n, \forall i$. Define $W_{H}$ to be the subset of $U$ where 
the rank of $e_{i j}$ in the Azumaya algebra is $h_{i j}$. Then $W_{H}$ is an irreducible component of $U$ and $U=\amalg_{H} W_{H}$.

Proof. The rank of an idempotent, in a sheaf of Azumaya algebras, is constant on connected components. Hence $W_{H}$ is a union of connected components. We consider the variety $P_{i}$ parametrizing homomorphisms $\prod_{j=1}^{m_{i}} \bar{k} \rightarrow M_{n}(\bar{k})$ where we $\operatorname{fix} \operatorname{rk}\left(e_{i j}\right)=h_{i j}$. Then this is a homogeneous space for $\operatorname{PGl}_{n}(\bar{k})$, $P_{i}=\left(\prod_{j=1}^{m_{i}} \mathrm{Gl}_{h_{i j}}(\bar{k})\right) \backslash \mathrm{Gl}_{n}(\bar{k})$, since all such homomorphisms are conjugate. $W_{\boldsymbol{H}}$ is the open subset of $\prod_{i} P_{i}$ where the corresponding subalgebras of $\boldsymbol{M}_{n}(\bar{k})$ generate $M_{n}(\bar{k})$. Hence $W_{H}$ is irreducible. $H$.

It is clear that $U=\amalg_{H} W_{H}$. Of course $W_{H}$ may well be empty for a given

This describes the set of minimal prime ideals in $\operatorname{spec}_{n} C$, which is homeomorphic to $U$; they are parametrized by the set $\mathscr{H}=\{H\}$ where $H$ runs through all sets of the type described in Theorem 2.4.

We need to describe the Galois action on these minimal primes. Fix an $i$; then $G$ acts on $\prod_{j=1}^{m_{i}} \bar{k}$ with fixed ring $l_{i}$. This induces an action of $G$ on the set $\mathscr{H}$. By 2.3 we deduce:

Theorem 2.5. The minimal primes of $\operatorname{spec}_{n} C$ are parametrized by the orbits of $G$ on $\mathscr{H}$, such that $W_{H}$ is nonempty for $H$ a representative of the orbit. We illustrate this for a small example:

Consider $C=\left(k(\sqrt{ } a) *_{k} k(\sqrt{ } b) *_{k} k(\sqrt{ } c)\right)_{2}$. In this case, $\mathscr{H}$ consists of tables

$$
H=\left(\begin{array}{ll}
h_{11} & h_{12} \\
h_{21} & h_{22} \\
h_{31} & h_{32}
\end{array}\right), \quad h_{i 1}+h_{i 2}=2 .
$$

If two different $h_{i j}$ 's are two, then it is clear that $W_{H}$ is empty. There are seven remaining possibilities

$$
\begin{gathered}
\left(\begin{array}{ll}
2 & 0 \\
1 & 1 \\
1 & 1
\end{array}\right),\left(\begin{array}{ll}
0 & 2 \\
1 & 1 \\
1 & 1
\end{array}\right),\left(\begin{array}{ll}
1 & 1 \\
2 & 0 \\
1 & 1
\end{array}\right),\left(\begin{array}{ll}
1 & 1 \\
0 & 2 \\
1 & 1
\end{array}\right) \\
\left(\begin{array}{ll}
1 & 1 \\
1 & 1 \\
2 & 0
\end{array}\right), \quad\left(\begin{array}{ll}
1 & 1 \\
1 & 1 \\
0 & 2
\end{array}\right),\left(\begin{array}{ll}
1 & 1 \\
1 & 1 \\
1 & 1
\end{array}\right) .
\end{gathered}
$$

It is clear that the first six give three G-orbits and the last is another G-orbit, so there are four minimal primes.

Now that we have begun to describe the minimal prime ideals of $\left(*_{k} L_{i}\right)_{n}=$ $C$, it is clear how we should modify our original requirements. We wish to find a division algebra $D$ of index $n$ over a field $K \supset k$ such that $K \otimes_{k} L_{i}$ embeds in $D$, that is, $L_{i}$ and $K$ are linearly disjoint in $D$. In this case, we write $n=m_{i} h_{i}$ where $m_{i}=\left[L_{i}: k\right]$ and $h_{i}$ is an integer. We find on passing to $D \otimes_{K} \bar{K}$ for $\bar{K}$ a separable closure of $K$ that such a point lies above the minimal prime corresponding to $H=\left\{h_{i j}: h_{i j}=h_{i}\right\}$, which is clearly $G$-invariant.

Theorem 2.6. Let $C=\left(*_{k} L_{i}\right)_{n}$ where $\left\{L_{i}: i=1, \ldots, m\right\}, 1<\left[L_{i}: k\right]=m_{i} \mid n$ and assume that either $m>2$ or $m_{i}>2$ for some $i$. Let $p$ be the minimal 
prime corresponding to $H=\left\{h_{i j}: h_{i j}=h_{i}=n / m_{i}\right\}$. Then $C / p$ is a domain, and its division algebra of fractions, $Q(C / p)$, is of index $n$.

Proof. Theorem 3.8 shows that for some field $K$ there is a central division $K$-algebra of index $n, D \supset K \supset k$ such that $L_{i} \otimes_{k} K$ embeds in $D$, and then Theorem 4.1 shows that there is a nonempty open subset of $\operatorname{spec}\left(C / p \otimes_{k} K\right)$ of points which map surjectively onto $D$.

Let $q$ be such a point and consider the localization $\left(C / p \otimes_{k} K\right)_{q}$ which is a local Azumaya algebra. On an open subset of $\operatorname{spec}\left(C / p \otimes_{k} K\right)$, we may assume that the center of $\left(C / p \otimes_{k} K\right)_{q}$ is regular, and so, of finite global dimension. The same holds for the Azumaya algebra $\left(C / p \otimes_{k} K\right)_{q}$, which must be a domain by Walker's theorem [12].

Hence $C / p \otimes_{k} K$ and $C / p$ must be domains. The remark about the index of $Q(C / p)$ also follows.

We call $Q(C / p)$ the division algebra coproduct of index $n$ of $\left\{L_{i}\right\}$ amalgamating $k$. We end this section by describing the elements of $Q(C / p)$ algebraic over $k$. First we note:

Theorem 2.7. Define $Q(C / p)$ as in the foregoing theorem. The center of $Q(C / p)$ is regular over $k$.

Proof. It is the function field of the variety $W_{H}$ (see Theorem 2.4) which was shown to be absolutely irreducible.

Finally, we have constructed a division algebra containing any given family of separable field extensions. It is of some interest to describe the complete set of algebraic field extensions of $k$, that lie in $Q(C / p)$. The next theorem does this, and the proof is a sketch which the interested reader may fill in.

The answer we obtain is independent of the P.I. degree, and coincides with the answer for the skew field coproduct (Theorem 9.3 of [10]). For consequences of this condition, one may look at Chapter 9 in loc. cit.

Theorem 2.8. Define $Q(C / p)$ as in Theorem 2.6. Then the algebraic extension $L \supset k$ embeds in $Q(C / p)$ if and only if $\operatorname{hcf}_{i j}\left[L_{i j}: L_{i}\right]=1$ where $L_{i} \otimes_{k} L \simeq$ $\prod_{j} L_{i j}$.

Proof. We consider $C \otimes_{k} L \simeq\left(*_{L}\left(L_{i} \otimes_{k} L\right)\right)_{n} \simeq\left(*_{L} \prod L_{i j}\right)_{n}$. We have the prime factor ring $C / p \otimes_{k} L$; it is prime because the center of $Q(C / p)$ is regular and hence $Q(C / p) \otimes_{k} L$ is a simple algebra. We wish to find its index. We begin by describing the ranks of the idempotents in $L_{i} \otimes_{k} L$ under the embeddings into $Q(C / p) \otimes_{k} L$.

Write $L_{i} \otimes_{k} L=\prod_{j} L_{i j}$ with corresponding idempotents $\left\{e_{i j}\right\}$, then the rank of $e_{i j}$ in $Q(C / p) \otimes_{k} L$ must be $n\left(\left[L_{i j}: L\right] /\left[L_{i}: k\right]\right)$.

If $\operatorname{hcf}\left[L_{i j}: L_{i}\right]=1$,

$$
\begin{aligned}
\operatorname{hcf}\left\{n\left[L_{i j}: L\right] /\left[L_{i}: k\right]\right\} & =\operatorname{hcf}\left\{n\left[L_{i j}: k\right] /\left[L_{i}: k\right][L: k]\right\} \\
& =\operatorname{hcf}\left\{n\left[L_{i j}: L_{i}\right] /[L: k]\right\}=n /[L: k]
\end{aligned}
$$

which implies that $Q(C / p) \otimes_{k} L \simeq M_{l}(S)$ for some simple algebra $S$ where $l=[L: k]$ and so $L$ embeds in $Q(C / p)$. Conversely, if $\operatorname{hcf}\left\{\left[L_{i j}: L_{i}\right]\right\}=$ $t>1$, then $[L: k]=l=s t$ for some integer $s$ and by Theorem 3.10 there is a division algebra $D^{\prime}$ of index $n / s$ over a regular extension of $L$ such that 
$L_{i j}$ embeds in $M_{r_{i j}}\left(D^{\prime}\right)$ where $r_{i j}=\left[L_{i j}: L_{i}\right] / t$. This implies that $\prod_{j} L_{i j}$ embeds in $M_{s}\left(D^{\prime}\right)$ so that the rank of $e_{i j}$ is $n\left(\left[L_{i j}: L\right] /\left[L_{i}: k\right]\right)$, and so the corresponding point of $\left(*_{k} L_{i}\right)$ lies above the prime $p$. It follows that we have a map from $(C / p) \otimes_{k} L$ to $M_{s}\left(D^{\prime}\right)$ inducing the correct map on $\prod_{j} L_{i j}$. Since a general point above $p$ gives an algebra of P.I. degree $n$, we may adjust this to a surjective map from $C / p \otimes L$ to $M_{s}\left(D^{\prime}\right)$. Another application of Walker's theorem allows us to conclude that $Q(C / p) \otimes_{k} L \simeq M_{s}(D)$ for some division algebra $D$, hence $L$ does not embed in $Q(C / p)$.

\section{GeOMETRIC METHODS}

Let $S$ be a central simple algebra over a field $k$ of dimension $n^{2}$. Since $S$ is a twisted form of $M_{n}(k)$, there is a corresponding cohomology class $\sigma \in H^{1}\left(G, \mathrm{PGl}_{n}(\bar{k})\right)$ where $G$ is the Galois group of the separable closure $\bar{k}$ over $k$. We shall be constructing a number of varieties associated to $S$ whose function fields have certain generic properties and we begin by recalling a number of facts about the Brauer-Severi variety. Our point of view will be that these varieties represent certain functors from field extensions of $k$ to the category of sets.

Consider the variety $\mathbf{P}^{n-1}$ defined over the field $k, \mathbf{P}^{n-1}(L)$ is the collection of 1-dimensional linear subspaces of $L^{n} \simeq L \otimes_{k} k^{n}$. By Morita equivalence, we may regard $\mathrm{P}^{n-1}(L)$ as the collection of left ideals of rank $n$ in $M_{n}(L) \simeq$ $L \otimes_{k} M_{n}(k)$. The first description gives the universal line subbundle $\mathscr{O}(-1)$ on $\mathbf{P}^{n-1}$ and this generates the Picard group over any field extension of $k$.

The algebraic group $\mathrm{PGl}_{n}$ has a natural action on $\mathbf{P}^{n-1}$ via the action of $\mathrm{Gl}_{n}$ of $k^{n}$ or equivalently on $\mathbf{P}^{n-1}$ via its action by conjugation on $M_{n}(k)$. We may define a twisted form $\mathbf{P}_{\sigma}^{n-1}$ defined over the field $k$ corresponding to the cohomology class $\sigma$ in $H^{1}\left(G, \operatorname{PGl}_{n}(\bar{k})\right)$. This is the Brauer-Severi variety of $S$. Galois descent gives that $\mathbf{P}_{\sigma}^{n-1}(L)$ is the set of left ideals of rank $n$ in $L \otimes_{k} S . \mathbf{P}_{\sigma}^{n-1}(L)$ is nonempty, therefore, if and only if $L \otimes_{k} S \simeq M_{n}(L)$, which means that $L$ is a splitting field for $S$ and hence $\sigma$. So, $\mathbf{P}_{\sigma}^{n-1} \times{ }_{k} L \simeq \mathbf{P}^{n-1} \times_{k} L$.

If $X$ is an irreducible variety over $k$, we define $k(X)$ to be its function field. We wish to know the kernel of the composite map $\operatorname{Br}(k) \rightarrow \operatorname{Br}(X) \rightarrow \operatorname{Br}(k(X))$ for a number of varieties $X$. If $X$ is a smooth variety, which will be the case in all our examples, the second map $\operatorname{Br}(X) \rightarrow \operatorname{Br}(k(X))$ is injective, therefore, it is enough to determine the kernel of $\operatorname{Br}(k) \rightarrow \operatorname{Br}(X)$. The following result [1] allows us to determine this.

Theorem 3.1. There is an exact sequence

$$
\operatorname{Pic}(X) \rightarrow H^{0}\left(G, \operatorname{Pic}\left(X \times_{k} \bar{k}\right)\right) \stackrel{\phi(X)}{\longrightarrow} \operatorname{Br}(k) \rightarrow \operatorname{Br}(X)
$$

which is functorial in the variety $X$.

The middle map $\phi(X)$ is well known for the Brauer-Severi variety. In this case, $\operatorname{Pic}\left(\mathbf{P}_{\sigma}^{n-1} \times_{k} \bar{k}\right) \simeq \operatorname{Pic}\left(\mathbf{P}^{n-1} \times_{k} \bar{k}\right)$ is isomorphic to $\mathbf{Z}$ with generator $\mathscr{O}(-1)$, and the following result holds.

Theorem 3.2. $\phi\left(\mathbf{P}_{\sigma}^{n-1}\right)(\mathscr{O}(-1))=[S]$. Therefore, the kernel of the map $\operatorname{Br}(k) \rightarrow$ $\operatorname{Br}\left(\mathbf{P}_{\sigma}^{n-1}\right)$ is the subgroup generated by $[S]$. The same holds for $\operatorname{Br}(k) \rightarrow$ $\operatorname{Br}\left(k\left(\mathbf{P}_{\sigma}^{n-1}\right)\right)$. 
Next, we discuss a mild generalization of $\mathbf{P}_{\sigma}^{n-1}$ briefly mentioned in Artin [1]. The algebraic group $\mathrm{PGl}_{n}$ acts on $\mathrm{Gr}\left(\begin{array}{l}n \\ m\end{array}\right)$, the Grassmannian space of $m$-dimensional subspaces of a vector space of dimension $n$. We consider the twisted form $\operatorname{Gr}\left(\begin{array}{c}m \\ n\end{array}\right)_{\sigma}$ corresponding to the image of $\sigma$ in

$$
H^{1}\left(G, \operatorname{Aut}\left(\operatorname{Gr}\left(\left(\begin{array}{l}
n \\
m
\end{array}\right) \times_{k} \bar{k}\right)\right) .\right.
$$

Since one may also regard $\operatorname{Gr}\left(\begin{array}{l}n \\ m\end{array}\right)(L)$ as the space of left ideals of rank $m n$ in $M_{n}(L)$, we see by Galois descent, that $\operatorname{Gr}\left(\begin{array}{c}n \\ m\end{array}\right)_{\sigma}(L)$ is the space of left ideals of rank $m n$ in $S \otimes_{k} L$. If $m$ is co-prime to $n$, then it follows that $\operatorname{Gr}\left(\begin{array}{l}n \\ m\end{array}\right)_{\sigma}(L)$ is nonempty if and only if $L$ is a splitting field for $S$. It is more interesting when $1 \neq t=\operatorname{hcf}\{n, m\}$, then $\operatorname{Gr}\left(\begin{array}{l}n \\ m\end{array}\right)_{\sigma}(L)$ is nonempty if and only if $S \otimes_{k} L \simeq$ $M_{s}\left(S^{\prime}\right)$ where $s=n / t$ and $S^{\prime}$ is a central simple $L$-algebra.

We may regard the function field of $\operatorname{Gr}\left(\begin{array}{l}n \\ m\end{array}\right)_{\sigma}$ as a generic partial splitting field of $S$. This construction has also been studied by A. Blanchet [3].

We consider the Picard group of $\operatorname{Gr}\left(\begin{array}{l}n \\ m\end{array}\right)_{\sigma} \times_{k} \bar{k} \simeq \operatorname{Gr}\left(\begin{array}{l}n \\ m\end{array}\right) \times{ }_{k} \bar{k}$. This is isomorphic to $\mathbf{Z}$ and this arises in the following way. Let $V \subset k^{n}$ be an $m$ dimensional subspace, then $\Lambda^{m} V \subset \Lambda^{m} k^{n}$ is a 1-dimensional subspace of the fixed vector space $\Lambda^{m} k^{n} \simeq k^{N}$ where $N=\left(\begin{array}{l}n \\ m\end{array}\right)$, the binomial coefficient. This defines a map $\mu: \operatorname{Gr}\left(\begin{array}{l}n \\ m\end{array}\right) \rightarrow \mathbf{P}^{N-1}$, the Plucker embedding, and this morphism induces an isomorphism on Picard groups. We wish to determine the kernel of the map from $\operatorname{Br}(k)$ to $\operatorname{Br}\left(\operatorname{Gr}\left(\begin{array}{l}n \\ m\end{array}\right)_{\sigma}\right)$, and so we wish to find a version of the map $\mu$ from $\operatorname{Gr}\left(\begin{array}{l}n \\ m\end{array}\right)_{\sigma}$ to some suitable Brauer-Severi variety. We consider some related algebra constructions. The following discussion is another point of view for $\S 4$ of Artin [1].

Given a partition $\alpha=\left(\alpha_{1}, \alpha_{2}, \ldots\right)$ of the integer $s$, where $\alpha_{1} \geq \alpha_{2} \geq \cdots$, there is a corresponding representation of $\mathrm{Gl}_{n}$; it is trivial if the partition has more than $n$ terms. We denote this representation by $\Sigma^{\alpha}\left(k^{n}\right)$. There is a corresponding algebra construction, $\Sigma^{\alpha}\left(M_{n}(k)\right)=\left(\Sigma^{\alpha}\left(k^{n}\right)\right)^{*} \otimes_{k} \Sigma^{\alpha}\left(k^{n}\right)$. This is not usually discussed on the grounds of its triviality since it is simply $\operatorname{End}_{k}\left(\Sigma^{\alpha}\left(k^{n}\right)\right)$. However, it gains a little interest from the observation that the action of $\mathrm{Gl}_{n}$ is actually an action of $\mathrm{PGl}_{n}$ and we may construct the associated twisted form, which we shall denote by $\operatorname{End}\left(\Sigma^{\alpha}\left(k^{n}\right)\right)_{\sigma}$. Artin's discussion shows that its class in $\operatorname{Br}(k)$ is $s[S]$. Our interest is in the case $\operatorname{End}\left(\Lambda^{m}\left(k^{n}\right)\right)_{\sigma}$.

We consider the Plucker embedding in terms related to the preceding paragraph. A left ideal of rank $m n$ in $M_{n}(k)$ is an embedding $\left(k^{n}\right)^{*} \otimes_{k} V \rightarrow$ $\left(k^{n}\right)^{*} \otimes_{k} k^{n}$ where $V$ is $k^{m}$. Applying $\Lambda^{m}$ gives an embedding $\Lambda^{m}\left(\left(k^{n}\right)^{*}\right) \otimes$ $\Lambda^{m}(V)=\Lambda^{m}\left(k^{n}\right)^{*} \otimes_{k} k \hookrightarrow \Lambda^{m}\left(\left(k^{n}\right)^{*}\right) \otimes_{k} \Lambda^{m}\left(k^{n}\right)$.

Galois descent along the co-cycle $\sigma$ gives a left ideal in $\operatorname{End}\left(\Lambda^{m}\left(k^{n}\right)\right)_{\sigma} \otimes_{k} L$ of rank $\left(\begin{array}{l}n \\ m\end{array}\right)$ associated to a left ideal of rank $m n$ in $S \otimes_{k} L$. So, the Plucker embedding gives by Galois descent, a map $\mu^{\prime}: \operatorname{Gr}\left(\begin{array}{c}n \\ m\end{array}\right)_{\sigma} \rightarrow \mathbf{P}\left(\Lambda^{m}\left(k^{n}\right)\right)_{\sigma}$ where $\mathbf{P}\left(\Lambda^{m}\left(k^{n}\right)\right)_{\sigma}$ is the Brauer-Severi variety of $\operatorname{End}\left(\Lambda^{m}\left(k^{n}\right)\right)_{\sigma}$.

The next theorem follows simply.

Theorem 3.3. The kernel of the map from $\operatorname{Br}(k)$ to $\operatorname{Br}\left(\mathrm{Gr}\left(\begin{array}{l}n \\ m\end{array}\right)_{\sigma}\right)$ is generated by $m[S]$, which is the image of $\mu^{\prime *}(\mathscr{O}(-1))$ under $\phi\left(\mathrm{Gr}\left(\begin{array}{l}n \\ m\end{array}\right)_{\sigma}\right)$.

Proof. By Theorem 3.1, it is enough to compute

$$
\phi\left(\operatorname{Gr}\left(\begin{array}{l}
n \\
m
\end{array}\right)_{\sigma}\right): H^{0}\left(G, \operatorname{Pic}\left(\operatorname{Gr}\left(\begin{array}{l}
n \\
m
\end{array}\right)_{\sigma} \times \bar{k}\right)\right) \rightarrow \operatorname{Br}(k) .
$$


We have the following commutative diagram:

$$
\begin{aligned}
& H^{0}\left(G, \operatorname{Pic}\left(\mathbf{P}\left(\Lambda^{m}\left(k^{n}\right)\right)_{\sigma} \times \bar{k}\right)\right) \longrightarrow \operatorname{Br}(k) \\
& H^{0}\left(G, \operatorname{Pic}\left(\operatorname{Gr}\left(\begin{array}{l}
n \\
m
\end{array}\right)_{\sigma} \times \bar{k}\right)\right) \longrightarrow \operatorname{Br}(k)
\end{aligned}
$$

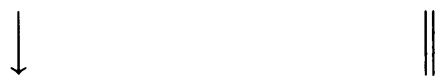

Since $\mathbf{P}\left(\Lambda^{m}\left(k^{n}\right)\right)_{\sigma}$ is the Brauer-Severi variety of an algebra of class $m[S]$ in the Brauer group and the map $\mu^{\prime}: \operatorname{Gr}\left(\begin{array}{l}n \\ m\end{array}\right)_{\sigma} \rightarrow \mathbf{P}\left(\Lambda^{m}\left(k^{n}\right)\right)_{\sigma}$ induces an isomorphism on Picard groups over $\bar{k}$, the results follows.

Given a variety $X$ defined over a finite separable field extension $L \supset k$, Weil defines a variety, $X \downarrow_{k}^{L}$, the restriction of $X$ from $L$ to $k$, defined over $k$ such that $X \downarrow_{k}^{L}(K)=X\left(L \otimes_{k} K\right)$. In this statement and in future similar statements we shall assume that $L \otimes_{k} K$ is a field since this simplifies our statements; it is not hard, but it is tedious, to keep track of the rank of idempotents in future statements.

Our interest in this construction arises from the following result.

Theorem 3.4. Assume that $L \supset k$ is a separable field extension of dimension $t$, that $S$ is a central simple $k$-algebra of dimension $n^{2}$, where $n=m t$ for integral $m$ and $t$. Assume that $K \otimes_{k} L$ is a field. Then $\left.\left(\operatorname{Gr}\left(\begin{array}{l}n \\ m\end{array}\right)_{\sigma}\right) \times_{k} L\right) \downarrow_{k}^{L}$ has a point in the field $K$ if and only if $L \otimes_{k} K$ embeds in $S \otimes_{k} K$.

Proof. $\left.\left(\operatorname{Gr}\left(\begin{array}{l}n \\ m\end{array}\right)\right)_{\sigma} \times_{k} L\right) \downarrow_{k}^{L}$ has a point in $K$ if and only if $\left(\operatorname{Gr}\left(\begin{array}{l}n \\ m\end{array}\right)_{\sigma} \times_{k} L\right)$ has a point in $L \otimes_{k} K$. This holds if and only if $S \otimes_{k} L \otimes_{k} K \simeq M_{t}\left(S^{\prime}\right)$ for some central simple $L \otimes_{k} K$-algebra $S^{\prime}$. But this holds if and only if $L \otimes_{k} K$ embeds in $S \otimes_{k} K$.

We may regard the function field of $\left(\operatorname{Gr}\left(\begin{array}{l}n \\ m\end{array}\right)_{\sigma} \times_{k} L\right) \downarrow_{k}^{L}$ as a generic field $F$ such that $F \otimes_{k} S$ contains $F \otimes_{k} L$. We wish to know that if $S$ is actually a division algebra then $F \otimes_{k} S$ is still a division algebra; in a subsequent paper [11], we shall actually prove this. Here we shall remain content with showing that the kernel of the map from $\operatorname{Br}(k)$ to $\operatorname{Br}\left(\left(\operatorname{Gr}\left(\begin{array}{l}n \\ m\end{array}\right)_{\sigma} \times L\right) \downarrow_{k}^{L}\right)$ is trivial, and so, if $S$ is a central division algebra of order and index $n$, it remains a division algebra. This is good enough to give a way to embed $L$ into a division algebra over some regular extension of $k$, as we wanted.

In order to compute the kernel of the map from $\operatorname{Br}(k)$ to $\operatorname{Br}\left(X \downarrow_{k}^{L}\right)$ for a variety $X$ over $L$, we need to be able to calculate the $G$-invariant invertible sheaves in $\operatorname{Pic}\left(X \downarrow_{k}^{L} \times \bar{k}\right)$. For this we need an explicit description of $X \downarrow_{k}^{L}$.

Let $H$ be the Galois group of $\bar{k}$ over $L$, let $\sigma_{i}: L \rightarrow L_{i} \subset \bar{k}$ for $i=1, \ldots, t$ be the embeddings of $L$ in $\bar{k}$ where $t$ is the dimension of $L$ over $k$. We form the variety $X^{\prime}=\prod_{i=1}^{t} X_{i} \times_{L_{i}} \bar{k}$ where $X_{i}$ is the variety over $L_{i}$ corresponding to $X$ over $L$ by the isomorphism $\sigma_{i}$. Then $G$ has an action on $X^{\prime}$, extending its action on $\bar{k}$ and $X^{\prime} \simeq\left(X \downarrow_{k}^{L}\right) \times_{k} \vec{k}$ which defines $X \downarrow_{k}^{L}$ as the fixed points under $G$. We calculate the Picard group of $X \downarrow_{k}^{L} \times \bar{k}$ from this at once. We say that a variety $X$ is geometrically rational if $X \times_{k} \bar{k}$ is rational.

Theorem 3.5. Let $X$ be a projective geometrically rational variety. Then

$$
\operatorname{Pic}\left(X \downarrow_{k}^{L} \times \bar{k}\right) \simeq \bigoplus_{i=1}^{t} \operatorname{Pic}\left(X \times_{L} \bar{k}\right) .
$$


$H^{0}\left(G, \operatorname{Pic}\left(X \downarrow_{k}^{L} \times \bar{k}\right)\right)$ is isomorphic to $H^{0}\left(H, \operatorname{Pic}\left(X \times_{L} \bar{k}\right)\right)$ under this isomorphism.

Proof. $X \downarrow_{k}^{L} \times \bar{k} \simeq \prod_{i=1}^{t} X_{i} \times_{L_{i}} \bar{k}$, so the first result follows since $\operatorname{Pic}(X \times Y)=$ $\operatorname{Pic}(X) \oplus \operatorname{Pic}(Y)$ if $X$ is rational. In fact, as $G$-module, $\operatorname{Pic}\left(X \downarrow_{k}^{L} \times \bar{k}\right) \simeq$ $\operatorname{Pic}\left(X \times_{L} \bar{k}\right) \otimes_{\mathbf{Z} H} \mathbf{Z} G$. The second result follows from this latter description.

Theorem 3.6. Let $S$ be a central simple algebra of dimension $n^{2}$. Let $n=m t$ for integers $m$ and $t$. Let $L \supset k$ be a separable field extension of dimension $t$ over $k$. Let $Y=\left(\operatorname{Gr}\left(\begin{array}{l}n \\ m\end{array}\right)_{\sigma} \times_{k} L\right) \downarrow_{k}^{L} . Y$ is an absolutely irreducible variety, so its function field $k(Y)$ is regular over $k . \operatorname{Br}(k)$ embeds in $\operatorname{Br}(k(Y))$, furthermore $L \otimes_{k} k(Y)$ embeds in $S \otimes_{k} k(Y)$. If $S=D$ is a division algebra whose order equals its index $n$, then $D \otimes_{k} k(Y)$ remains a division algebra.

Proof. There is a natural map from $\operatorname{Gr}\left(\begin{array}{l}n \\ m\end{array}\right)_{\sigma}$ to $\left(\operatorname{Gr}\left(\begin{array}{l}n \\ m\end{array}\right)_{\sigma} \times_{k} L\right) \downarrow_{k}^{L}$ given by $\left(\operatorname{Gr}\left(\begin{array}{l}n \\ m\end{array}\right)_{\sigma} \times_{k} L\right)_{i} \simeq \operatorname{Gr}\left(\begin{array}{l}n \\ m\end{array}\right)_{\sigma} \times L_{i}$. Over $\bar{k}$, this becomes the diagonal map from $\operatorname{Gr}\left(\begin{array}{l}n \\ m\end{array}\right)$ to $\prod_{i=1}^{t} \operatorname{Gr}\left(\begin{array}{l}n \\ m\end{array}\right)$. The $G$-invariant line bundles on $\prod_{i=1} \operatorname{Gr}\left(\begin{array}{l}n \\ m\end{array}\right)$ for the action giving descent to $\left(\operatorname{Gr}\left(\begin{array}{l}n \\ m\end{array}\right)_{\sigma} \times L\right) \downarrow_{k}^{L}$ are generated by $\mathscr{O}(-1) \times \cdots \times \mathscr{O}(-1)$, and this pulls back to $\mathscr{O}(-t)$ on $\operatorname{Gr}\left(\begin{array}{l}n \\ m\end{array}\right)$ which is $\operatorname{Gr}\left(\begin{array}{l}n \\ m\end{array}\right)_{\sigma} \times \bar{k}$. By Theorem 3.3, the image of this in $\operatorname{Br}(k)$ is $m t[S]$, and the same holds for the generator of the $G$-invariant invertible sheaves on $\left(\operatorname{Gr}\left(\begin{array}{l}n \\ m\end{array}\right)_{\sigma} \times L\right) \downarrow_{k}^{L}$ by functoriality. But if $n=m t$, this class is trivial. It follows that in this case $\operatorname{Br}(k)$ embeds in $\operatorname{Br}(Y)$ and hence $\operatorname{Br}(k(Y))$ where $Y=\left(\operatorname{Gr}\left(\begin{array}{l}n \\ m\end{array}\right)_{\sigma} \times L\right) \downarrow_{k}^{L}$. The last sentence follows since the index is always at least the order, and can only drop under extension.

In a subsequent paper, [11] we will remove the flaw from this theorem by showing that the index always remains constant under this construction. One consequence of such a theorem is that for every division algebra $D$ over $k$, there is an extension field $F$ such that $D \otimes_{k} F$ is a cyclic division algebra.

We give some simple consequences of Theorem 3.6.

Theorem 3.7. Let $D$ be a division algebra of index and order $n$. Let $\left\{L_{i}: i \in I\right\}$ be a possibly infinite set of separable extensions of $k$ such that the dimension of $L_{i}$ over $k$ divides $n$. Then there exists a regular field extension $F$ of $k$ such that $D \otimes_{k} F$ is a division algebra and $L_{i} \otimes_{k} F$ embeds in $D \otimes_{k} F$. Also, we may ensure that $\operatorname{Br}(k)$ embeds in $\operatorname{Br}(F)$.

Proof. Apply transfinite induction and Theorem 3.6.

Theorem 3.8. Let $\left\{L_{i}: i \in I\right\}$ be a family of separable field extensions of $k$ dimension dividing $n$. Then there exists a regular field extension $F$ of $k$ and a central division $F$-algebra $D$ of index and order $n$ such that $L_{i} \otimes_{k} F$ embeds in $D$ for each $i$. Further, we may ensure that $\operatorname{Br}(k)$ embeds in $\operatorname{Br}(F)$.

Proof. First, set $D_{0}$ to be the generic division algebra of index $n$. Its center, $C$, is unirational and hence regular over $k$ and $\operatorname{Br}(k)$ embeds in $\operatorname{Br}(C)$, so each $L_{i} \otimes_{k} C$ is a field. Now apply Theorem 3.7.

It has been a question for some time whether two central division algebras over a field $F$ having the same collection of subfields must generate the same subgroup of the Brauer group. We can do worse than that.

Theorem 3.9. There exists a field $F$ and two division algebras of index $n, D$ and $E$, such that the subgroup of the Brauer group generated by $D$ and $E$ is 
isomorphic to $C_{n} \times C_{n}$, and every field extension of $F$ of dimension dividing $n$ embeds in both $D$ and $E$.

Proof. We find some field $k$ of characteristic 0 having two division algebras $D_{0}, E_{0}$ of index $n$ such that their classes generate a subgroup isomorphic to $C_{n} \times C_{n}$ in $\operatorname{Br}(k)$. We apply Theorem 3.8 alternately to $D_{0}$ and $E_{0}$ and take the union.

By being careful about our unions, it is possible to find a field $F$ containing a given field $k$ such that $\operatorname{Br}(k)$ embeds in $\operatorname{Br}(F)$ and every central division algebra over $F$ contains any field extension of $F$ of dimension dividing the index of the division algebra. We leave this as an exercise for a long-minded reader.

Finally, we prove the technical result we needed in $\S 2$ to describe the commutative algebraic subfields of the division algebra coproduct of index $n$.

Theorem 3.10. Consider $\left\{\left(L_{i}, n_{i}\right), i \in I, L_{i}\right.$ a finite separable extension of $k$ of dimension dividing $\left.n n_{i}\right\}$. Then there exists a regular field extension $F$ of $k$, and a central division algebra $D \supset F$ of index $n$ such that $L_{i} \otimes_{k} F$ embeds in $M_{n_{i}}(D)$. Further, we may insure that $\operatorname{Br}(k)$ embeds in $\operatorname{Br}(F)$.

Proof. This is proved in the same way as Theorem 3.8 using a generalized version of Theorem 3.7. We leave the details to the reader.

\section{GeNERATORS FOR DIVISION ALGEBRAS}

Theorem 4.1. Let $S$ be a central simple algebra over an infinite field $k$, and let $E$ and $F$ be simple separable subalgebras. Let $[S: k]=n^{2},[E: k]=e$, and $[F: k]=f$. Then there exist conjugates $E^{\prime}$ and $F^{\prime}$ of $E$ and $F$ respectively that generate $S$ over $k$ if $e, f \geq 2$ and one of them is larger than 2. If $e=f=2$, then conjugates of $E$ and $F$ generate $S$ if and only if $n=2$.

Proof. We refer the reader to Kac [6] for undefined terms in the following proof. We deal first with the case where $E$ and $F$ are separable field extensions in the division algebra $D$. Over the algebraic closure $\bar{k}$ of $k$, we are considering copies of $\bar{k}^{e}$ and $\bar{k}^{f}$ embedded in $M_{n}(\bar{k})$ so that minimal idempotents in $\overline{\bar{k}}^{e}$ all have rank $n / e$ and minimal idempotents in $\bar{k}^{f}$ have rank $n / f$. This determines a collection of $e+f$ subspaces of $k^{n}$ by taking the images of the minimal idempotents. The dimension vector of this collection is

$$
(n, \underbrace{\frac{n}{e}, \ldots, \frac{n}{e}}_{e \text { copies }}, \underbrace{\frac{n}{f}, \ldots, \frac{n}{f}}_{f \text { copies }}) \text {. }
$$

Conversely, given a collection of subspaces in general position of this dimension vector, the $e$ subspaces of dimension $n / e$ will be linearly independent and determine an embedding of $\overline{\bar{k}}^{e}$ in $M_{n}(\overline{\bar{k}})$ via the projections on the subspaces. Similarly, we have an embedding of $\overline{\bar{k}}^{d}$ in $M_{n}(\overline{\bar{k}})$. The endomorphism ring of this collection of subspaces will be the centralizer of the algebra generated by $\overline{\bar{k}}^{e}$ and $\overline{\bar{k}}^{f}$.

We may apply a theorem of Kac to determine this endomorphism ring. If one of $e$ and $f$ is greater than 2, the corresponding dimension vector is a root 
for the associated quadratic form and lies in the fundamental region for the action of the Weyl group of the quadratic form on the Tits cone. Moreover it is not isotropic so the endomorphism ring of a generic collection of subspaces is $\bar{k}$. This implies that the centralizer of the subalgebra generated by $E$ and $F$ in general position in $D$ is $k$ since the $k$-points of $D^{*} \times D^{*}$ are dense in the $\bar{k}$-points. Hence these $E$ and $F$ generate $D$.

Note that if $e=f=2$, then we have to consider representations of the 4-subspace quiver of dimension vector

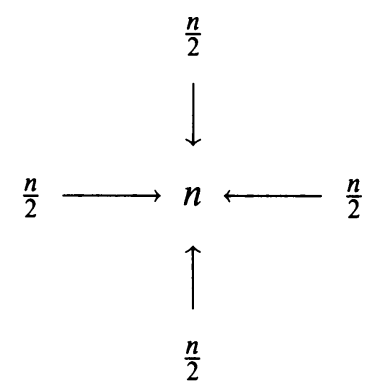

This is a root in the fundamental chamber, which in this case is isotropic. There is no representation of trivial endomorphism ring, except when $n=2$, which agrees with our previous discussion.

Next we deal with arbitrary simple separable algebras. All pairs of central simple algebras contain a pair of commutative separable algebras for which we already know the result, except in the case of two quaternion algebras, or a quaternion algebra and a quadratic extension; so it is enough to deal with the case where $E$ is a central quaternion algebra and $F$ a quadratic extension.

A $\bar{k}$-point determines an embedding of $M_{2}(\bar{k})$ and $\bar{k} \times \bar{k}$ in $M_{n}(\bar{k})$. Then the idempotents $e_{11}, e_{22}$ in $M_{2}(\bar{k})$ and the idempotents in $\bar{k} \times \bar{k}$ determine 4 subspaces of dimension $\frac{n}{2}$ in $\bar{k}^{n}$. Further, $e_{12}$ determines a map from the first to the second subspace. Thus we have a representation of the quiver:

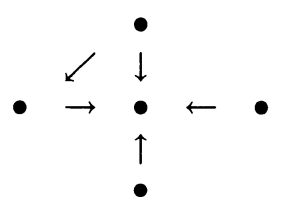

of dimension vector

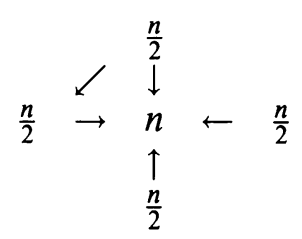

Conversely, given a general representation of this dimension vector, the first two subspaces will be linearly independent and the arrow between them will be represented by an isomorphism so that the projections on the subspaces together with this isomorphism and its inverse determine an embedding of $M_{2}(\bar{k})$ into $M_{n}(\bar{k})$; further, the second pair of subspaces will also be linearly independent which determine an embedding of $\overline{\bar{k}}^{2}$ into $M_{n}(\overline{\bar{k}})$. 
The endomorphism ring of this representation will be the centralizer of these subalgebras. However the dimension vector lies in the fundamental chamber, and is not isotropic, so by Kac [6], a general representation has endomorphism ring $\bar{k}$. Again, we deduce that a good pair of conjugates of $E$ and $F$ generate D.

Since every pair of simple separable algebras contains either a pair of commutative separable algebras or else a quaternion algebra and a quadratic extension we have dealt with the case where $S=D$, a division algebra.

We pass to the opposite extreme. If we have embeddings of $M_{a}\left(\bar{k}^{b}\right)$ and $M_{c}\left(\overline{\bar{k}}^{d}\right)$ into $M_{n}(\overline{\bar{k}})$ such that the ranks of the central idempotents in each separable algebra are constant, then this arises by field extension from the previous case (possibly after a change of algebraically closed field).

Since a general pair of conjugates of $E$ and $F$ generate $D$ and the dimension of the algebra generated is constant and maximal on an open subset, we deduce that we may find conjugates of $M_{a}\left(\overline{\bar{k}}^{b}\right)$ and $M_{c}\left(\overline{\bar{k}}^{d}\right)$ which generate $M_{n}(\overline{\bar{k}})$.

Finally, in the general case, the $k$-points of $S^{*} \times S^{*}$ are dense in the $\bar{k}$ points. Hence, there is a $k$-point where the dimension of the algebra generated is maximal and so there are conjugates of $E$ and $F$ which generate $S$.

We will use this point of view to show that a similar result cannot hold for semisimple subalgebras of a central simple algebra.

We consider $k^{2}$ and $k^{3}$ embedded in $M_{4}(k)$ so that the minimal idempotents have ranks $(3,1)$ and $(2,1,1)$ in the two cases. As in the preceding discussion, we are looking at a collection of five subspaces of $k^{4}$ with dimension vector $(4,3,1,2,1,1)$. One may show that if the subspaces are in general position this decomposes as a direct sum of a collection of subspaces of dimension vector $(1,1,0,1,0,0)$ and $(3,2,1,1,1,1)$. From this one may prove that the subring generated in general position by a copy of $k^{2}$ and a copy of $k^{3}$ having these ranks on idempotents is $k \times M_{3}(k)$.

\section{REFERENCES}

1. M. Artin (notes by A. Verschoren), Brauer-Severi varieties, Lecture Notes in Math., vol. 917, Springer-Verlag, New York, 1981.

2. G. Berman, Coproducts and some universal ring constructions, Trans. Amer. Math. Soc. 200 (1974), 33-88.

3. A. Blanchet (preprint), University of Austin, Texas.

4. B. Fein, Embedding rational division algebras, Proc. Amer. Math. Soc. 32 (1972), 427-429.

5. R. Hartshorne, Algebraic geometry, Graduate Texts in Math., no. 52, Springer-Verlag, New York, 1977.

6. V. Kac, Infinite root systems representations of graphs and invariant theory. II, J. Algebra 78 (1982), 141-162.

7. S. Lichtenbaum, The period-index problem for elliptic curves, Amer. J. Math. 90 (1968), 1209-1223.

8. J. S. Milne, Étale cohomology, Princeton Univ. Press, Princeton, N.J., 1980.

9. C. Procesi, Rings with a polynomial identity, Dekker, New York, 1973.

10. A. Schofield, Representations of rings over skew fields, London Math. Soc. Lecture Notes Ser. 92, Cambridge Univ. Press, Cambridge, 1985. 
11. A. Schofield and M. van den Bergh, The index of a Brauer class on a Brauer-Severi variety, preprint.

12. R. Walker, Local rings and normalizing sets of elements, Proc. London Math. Soc. (3) 24 (1972), 27-45.

Department of Mathematics, Yale University, New Haven, Connecticut 06520

Department of Mathematics and Computer Science, University of ANTwerp, UIA, ANTWERP 2610, BELGIUM

Current address (Michel Van den Bergh): Institut de Recherche Mathématique Avancée, Université Louis Pasteur et CNRS, 7, Rue René-Descartes, 67084 Strasbourg Cedex, France

School of Mathematics, University of Bristol, University Walk, Bristol B58 $1 \mathrm{JW}$, ENGLAND 